\title{
Relationship between tooth value and skin color in patients visiting Royal Medical Services clinics of Jordan
}

\author{
Hind Fahed Al-Nsour ${ }^{1}$, Tamara Trad Al-Zoubi², Ali Salaheddin Al-Rimawi ${ }^{3}$
}

${ }^{1}$ BDS, Jordanian Board in Pediatric Dentistry, Senior Specialist at the Pediatric Dentistry Department, King Hussein Medical Center, Royal Medical Services, Amman, Jordan

${ }^{2}$ BDS, Jordanian Board in Conservative Dentistry, Specialist at the Conservative Dentistry Department, King Hussein Medical Center, Royal Medical Services, Amman, Jordan

${ }^{3}$ BDS, Jordanian Board in Endodontics, Specialist at the Endodontic Department, King Hussein Medical Center, Royal Medical Services, Amman, Jordan

\section{Type of article: Original}

\begin{abstract}
Background: Facial and dental aesthetics are becoming of great concern for patients, especially for the younger generation. Tooth color matching and selection is considered a vital element in order to create an attractive beautiful smile. The importance of tooth color matching is to adjust tooth colors to obtain a good match with adjacent teeth and the skin. This is considered a challenge especially if adjacent teeth were lost or records of patients' teeth color were not available.

Objective: The aim of this study was to determine the relationship between skin complexion and tooth value in a Jordanian population.

Methods: A cross-sectional study of 520 individuals who were randomly selected and belonging to different age groups, ranging from 15-65 years, with equal sex distribution. These patients visited the dental clinics at different Jordanian military hospitals. The study was carried out over a period of one year from 2015 to 2016. The randomly selected sample was examined by one researcher. Shade of the middle third of the labial surface of central incisor was determined visually using VITA tooth guide, 3D-MASTER shade guide. Tooth shades were divided into two categories according to their value. The skin complexion was identified using Fitzpatrick skin type test, and was categorized into two categories (fair and dark). Data were statistically analyzed using Epi Info version 6.

Results: A statistically significant tooth shade value difference was discovered among subjects of different skin color $(\mathrm{p}<0.0001)$. Individuals with dark skin tend to have lighter teeth, while individuals with light skin tend to have darker teeth. Out of the 304 participants that were with dark complexion $274(90 \%)$ have light teeth, and only $30(10 \%)$ were with dark teeth. Out of the 216 participants with light complexion $172(80 \%)$ have dark teeth and only $44(20 \%)$ have light teeth.

Conclusion: Within the limitation of this study, there was an inverse relation between skin complexion and tooth value among a Jordanian population. Thus, considering the skin complexion when choosing tooth shades is helpful in achieving a pleasing good-looking smile, yet further investigations in this field must be carried out.

Keywords: Fitzpatrick scale, Skin complexion, Tooth value
\end{abstract}

\section{Introduction}

One of the reasons why patients visit dental clinics is to esthetically enhance and improve their smile. The majority of people think that brighter and lighter teeth are associated with more attractive smile, but in order to create a beautiful natural smile, one should not solely focus on the smile or the teeth alone, but it's harmony with the whole face should be considered so that it does not look absurd, or standout in an ugly way. To have a successful esthetic

\section{Corresponding author:}

Dr. Hind Fahed Al Nsour, Royal Medical Services, Amman, Jordan.

Tel.: +962.795050700, Email: nsourhind1@gmail.com

Received: January 10, 2018, Accepted: March 01, 2018, Published: March 2018

iThenticate screening: March 06, 2018, English editing: March 13, 2018, Quality control: March 15, 2018

This article has been reviewed / commented by three experts

(C) 2018 The Authors. This is an open access article under the terms of the Creative Commons Attribution-NonCommercialNoDerivs License, which permits use and distribution in any medium, provided the original work is properly cited, the use is non-commercial and no modifications or adaptations are made. 
smile that looks natural and attractive, certain determinants must be correctly chosen. The color of the restoration or the tooth is one of them. Tooth color is considered the most important factor of patients' perceptions of dental attractiveness (1). The whiter the teeth the prettier the smile, yet this mistaken perception of patients' dental attractiveness is not the only challenge a dentist has to overcome, but its harmony with its surroundings makes this procedure more challenging. Tooth shade or tooth color should be in harmony or in certain relationship with the skin complexion of the face (2). Color is a subjective and objective complex phenomenon that many systems tried to describe; one of these systems which is used widely in dentistry is the Munsell System $(3,4)$. According to this system, there are three parameters of color: hue, chroma, and value. Hue is the color appearance parameter, it is synonymous with the term color (red, blue, green, etc.) or the color produced by a specific light wave length acting on the retina. Chroma is the saturation or the intensity of the color, while value is the relative lightness or darkness of the color (5). A light tooth has high value, and a dark tooth has a low value. Value is considered the most important of the three parameters in shade selection, since a small variation in chroma and hue will not be noticeable if value blends (6). Value differences are easily detected even by untrained eyes (7), and can be easily noticed from near or far distances, but as the viewing distance increases, the differences in hue and chroma become less noticeable (7). There are many factors that affect tooth shade such as age and gender $(2,8)$. With age, teeth become darker and yellower $(9,10)$, and females tend to have lighter teeth than males $(9,11)$. Skin complexion is the visual assessment of skin color and tone. It can be affected by many factors as age, genetics, sun exposure and ultraviolet rays, hormones, vitamins and nutritional deficiency, acne and improper skin care, or the use of wrong cosmetics (12). Several studies were carried out to determine the relationship between skin complexion and tooth shade (10, 11,13-15). Some of which, found an inverse relationship between the two parameters while others found no existing relationship between them. Yet, further research is needed to be conducted among Jordanians to consider all elements that might affect teeth shade selection in order to enhance the good looking and natural appearance of the individuals' smiles. The rationale of this study is to determine the relationship between skin complexion and tooth color when designing and planning removable or fixed restorations. This study will be a valuable aid for dentists when restoring patients' teeth in order to achieve an aesthetic restoration that is in harmony with the surroundings, thus enhancing the natural beauty of their smile.

\section{Material and Methods}

\subsection{Research Design and Setting}

This was an analytical cross-sectional study, conducted at the Royal Medical Services dental clinics in different cities across Jordan to make sure that this sample is a true representation of the Jordanian population and its distribution. It was done over one year, from 2015 to 2016, in which a total of 520 patients belonging to different age groups ranging from 15 to 65 years were examined, with equal sex distribution to avoid gender bias. Before the study was conducted, written informed consent was taken from all participants. In addition, ethical approval was obtained from the Human Research Ethics Committee at Royal Medical Services.

\subsection{Sampling}

Our sample consists of 520 patients visiting the Royal Medical services dental clinics for dental care. This sample is greater than that which was identified by the Sample Size Calculator by Raosoft, Inc. (385 patients), with a marginal error of 5\% and confidence level of $95 \%$ (16). Thus ensuring minimal marginal error less than 5\%. Non-probability consecutive sampling technique was used to select the sample.

\subsection{Measurement Tools}

Tooth shade selection was done visually using VITA 3D-MASTER tooth shade guide. One of the main reasons of using VITA 3D-MASTER tooth shade guide was its accuracy. According to Funda Bayindir et al., this shade guide has the lowest coverage errors compared to Vita Lumin or Chromascope, and its coverage error did not differ significantly when all three shade guide systems were used. Clinically this system provides high potential for good visual shade match, and using it separately is as effective as using a combination of all 3 systems together (17). This system measures the value of the tooth, which is the color parameter that this study is addressing, and using it makes data classification easier, as it is an objective numeric measurement of the value and the color of the tooth (17), moreover, the system is readily available at our dental clinics. There are many ways to determine skin type or complexion, such as visual inspection, objective measurement (e.g. spectrometer), and self-report (Fitzpatrick scale) (18). The Fitzpatrick scale is still a recognized tool for dermatological research of skin pigmentation (19). It can translate the skin type into numbers, thus eliminating observers' perception error. This scale has identified six different numerical classification schemes for human skin color, ranging from Type I being the fairest with the lowest scores to Type VI being the darkest with the highest scores $(20,21)$. This numerical classification was based 
on a questionnaire related to participants' reaction to sun exposure and genetics. The response to each question was measured on a scale of $0-4$, then the responses were added to get the final score that corresponded to Fitzpatrick skin type (22). The following list shows the six categories of the Fitzpatrick scale according to the Skin Cancer Foundation (23):

- $\quad$ Type I (scores 0-6) always burns, never tans, blue, gray eyes, pale white, freckles, blond or red hair.

- Type II (scores 7-12) usually burns, tans minimally, blue, green, or hazel eyes, blond, brown or red hair, white complexion.

- $\quad$ Type III (scores 13-18) sometimes mild burn, tans uniformly, cream white, yellowish, any hair color.

- $\quad$ Type IV (scores 19-24) burns minimally, always tans well, light brown or olive, dark brown to black hair.

- $\quad$ Type V (scores 25-30) very rarely burns, tans very easily with brown complexion.

- Type VI (scores 30+) never burns, always tans with deeply pigmented dark brown to darkest brown complexion.

\subsection{Data Collection}

Patients were selected according to inclusion and exclusion criteria after proper history taking and clinical examination. The inclusion criteria were any subject, above the age of 15 years, with fully erupted maxillary anterior teeth, that have not been endodontically treated, restored, carious, or with intrinsic stains or developmental defects. Participants should be without any dermatological diseases, or undergoing any dermatological treatment. The exclusion criteria were subjects with history of bleaching, abnormality in tooth development, intrinsic staining, restored or endodontically treated maxillary central incisors and skin pigmented diseases. After the initial inclusion, tooth polishing procedure was done to the patients before selecting the tooth shade to remove any extrinsic stains. Special considerations were taken for female subjects as they were asked to remove lipstick or lip gloss at the time of clinical examination and data collection. Patients were asked to wear a neutral color, and a gray apron was put over their clothes at the time of tooth shade selection to avoid the effect of their colorful clothes on tooth color perception of the examiner, thus affecting tooth color selection procedure. Data was collected by one researcher to ensure standardization

\subsection{Tooth Shade and Skin Type selection}

Shade reading was taken after passing an Ishihara color-blindness test (24), and tooth shade was determined using VITA 3D-MASTER tooth shade guide, following manufacture instructions (25). The lightness group (L) was first determined, followed by chroma $(\mathrm{C})$, and finally the hue $(\mathrm{H})$. This order better matches the capabilities of the human eye, and corresponds to the importance of the three color elements in obtaining an accurate shade match (25, 26). One of the main principles that were followed in shade selection was that patients were viewed at eye level so that the retina's most color-sensitive part was used (27). The clinician's position was at an arm's length from the subject, since this distance of $25-33 \mathrm{~cm}$ is the standard reading distance that is used by ophthalmologist in visual acuity tests for near vision (28). Shade comparison was made on clean teeth under different lighting conditions as incandescent and fluorescent lightening, and the color was confirmed under natural daylight after initial selection. Shade comparison was made at the start of the patient's visit to avoid dehydration of the tooth; as dehydrated teeth appear lighter. Brightly colored clothing were draped and lipstick was removed as their colors might affect perception of the examiner. Shade comparison should be made quickly without staring at a tooth for more than 5 seconds, since our eyes might become accommodated to different colors, thus affecting the tooth color selection procedure. Tooth shade guide was placed under the lip directly, next and parallel to the tooth being matched, and if possible, it should be in the same plane with the tooth, not behind it or it will appear darker, nor in front of it or it will appear lighter (28). Immediately before shade comparison, the eye was focused on a gray-blue surface in order to balance all the color sensors of the retina and resolve the afterimage if it occurred. A spectrometer randomized control trial was done to ensure the accuracy of our results. The upper central incisor was used to determine tooth shade as a representative of individual's natural tooth color. The middle third of the labial surface was used to determine the correct shade. It was found to be the best area to illustrate tooth shade; since the incisal third of the tooth is translucent and is affected by the background, while the cervical third is modified by the scattered light from the gingiva (29). After identifying the tooth color of all participants, data were categorized into two groups following VITA 3D-MASTER tooth shade guide, where value 1 and 2 were put in one category which is the category of light teeth, and value 3 and 4 were considered as another category of dark teeth. In this study there were no participants with teeth value of 5 as illustrated in Table 1. The participants were examined by one examiner and based on a questionnaire, answers were recorded. Certain unclear tricky questions were clarified to people with colored skin in order to be able to accurately describe their response to the sun. Questions related to sun sensitivity in the Fitzpatrick skin test were rephrased by the examiner. Terms such as 'skin was tanned' or 'got burnt' were replaced by specific 
descriptions such as skin became darker, itchy or irritated, since using the Fitzpatrick skin type test without clarifying these questions results in little relationship between the skin-type categories and sun-reactivity (30). Using specific descriptors such as skin irritation, tenderness, itching, or skin becoming darker, ensure more accurate results (31). These answers and examination results were collected, and Fitzpatrick skin type of each candidate was identified. Based on Fitzpatrick skin type, we categorized patients' skin complexion into two categories. The fair skin category with Fitzpatrick skin type I, II and III, and the dark skin category with Fitzpatrick skin type IV and above. This is to ensure more or less equal distribution of the collected data, since the most common Fitzpatrick skin types in our study were type III and IV. Data gathered were statistically analyzed, using Epi Info (Version 6).

\section{Results}

Our sample was composed of 520 participants with equal sex distribution; 260 were males and 260 were females, with an average age of 30.8 years (mode 30 years), of different socioeconomic status. A significant relation was found between dark skin complexion category and the light tooth (tooth with high value category), and between light skin complexion category and the dark tooth (tooth with low value category) $(\mathrm{p}<0.0001)$. As per Table 1 , it is clear that the most common tooth value within Jordanians was tooth with value 2, with 286 patients (55\%), followed by tooth value 3 with 164 patients (32\%), then tooth value 4 with 38 (7\%). Patients with Light tooth category (Tooth values 1 and 2) were 318 patients $(61.15 \%$ ), against $38.85 \%$ for patients with Dark tooth category (Tooth values 3 and 4). According to Table 2, the most common Fitzpatrick skin type was type IV with 250 participants (48.08\%), then Fitzpatrick skin type III with 154 participants (29\%). The least skin types within Jordanians were types I and VI. Out of the 520 participants, 216 patients $(42 \%)$ were with fair complexion which were composed of Fitzpatrick skin I, II and III, and 304 out of the 520 participants (58\%) were with dark complexion. According to the results in this study, and as shown in Table 3, out of the 304 Jordanians that were categorized with dark complexion, 274 patients were with light and only 30 were with dark teeth. Whereas, out of the 216 participants who were considered with light complexion, 172 had dark teeth and only 44 had light teeth.

Table 1. Participants' distribution according to the value of their teeth.

\begin{tabular}{|l|l|l|l|}
\hline Category & Tooth value & Count vs. tooth value, $\mathrm{n}(\%)$ & Count vs. tooth shade, $\mathrm{n}(\%)$ \\
\hline \multirow{2}{*}{ Light Tooth } & 1 & $32(6.15 \%)$ & $318(61.15 \%)$ \\
\cline { 2 - 4 } & 2 & $286(55.00 \%)$ & \\
\hline \multirow{2}{*}{ Dark Tooth } & 3 & $164(31.54 \%$ & $202(38.85 \%)$ \\
\cline { 2 - 4 } & 4 & $38(7.31 \%)$ & $520(100 \%)$ \\
\hline Total & & $520(100 \%)$ & \\
\hline
\end{tabular}

Table 2. Participants' distribution according to the skin type (Fitzpatrick).

\begin{tabular}{|l|l|l|l|}
\hline Category & Fitzpatrick skin type & Count vs. skin type, n (\%) & Count vs. complexion, n (\%) \\
\hline Light Complexion Skin & I & $2(0.38 \%)$ & \multirow{3}{*}{ 216 (41.5\%) } \\
\cline { 2 - 3 } & II & $60(11.54 \%)$ & \\
\cline { 2 - 3 } & III & $154(29.62 \%)$ & \multirow{3}{*}{$304(58.5 \%)$} \\
\hline \multirow{2}{*}{ Dark Complexion Skin } & IV & $250(48.08 \%)$ & \\
\cline { 2 - 3 } & V & $52(10.00 \%)$ & \multirow{3}{*}{$520(100 \%)$} \\
\cline { 2 - 3 } & VI & $520(100 \%)$ & \\
\hline Total & & & \\
\hline
\end{tabular}

Table 3. Relation of the two categories of skin complexion to the two categories of tooth shade value $(\mathrm{p}<0.0001)$.

\begin{tabular}{|l|l|l|l|}
\hline Category & Light Teeth & Dark Teeth & Total \\
\hline Dark Complexion Skin & 274 & 30 & 304 \\
\hline Light Complexion Skin & 44 & 172 & 216 \\
\hline Total & 318 & 202 & 520 \\
\hline
\end{tabular}

\section{Discussion}

The goal of this study was to identify a parameter that could be useful when selecting the tooth shade for designing and planning removable dentures, or for full mouth reconstruction. This is considered of great importance when we are planning to create a natural looking smile especially when adjacent teeth are lost, and no previous records of teeth color are available. Creating a perfect harmonic scheme between colors is highly essential in the world of art and design. The rules of color harmony apply equally well in designing an esthetically good looking smile in terms 
of visual perception. Therefore, harmonizing the background color of the skin with the foreground color of teeth is considered an important element in smile design and facial beauty. Skin complexion varies from one person to another, and the Fitzpatrick scale sums them up in categories. Yet little information was mentioned in literature on Jordanians' skin types, but according to our results, most of the Jordanian population are with skin type IV and III. This result agreed with a study which was carried out on a Northern Jordanian population by Omari et al. (32). The results of this study established an inverse relation between skin complexion and tooth value. Persons with medium dark and dark skin were more likely to have teeth in the high value category than persons with medium fair and fair skin, and vice versa. These results agreed with the findings of many researchers such as Jahangiri L et al. (10), and Suneel V. K. (14) who found an inverse relation between tooth shade and skin complexion, but disagreed with other researchers such as Esan TA et al. (11), and Al-Dwairi Z (15) who found no relation between tooth shade and skin complexion. This could be due to the difference in population selection, or might be due to using different shade guides. In our study, we used VITA 3D Master which, according to the manufacturer, covers the whole color space and reflects distribution of tooth shade in nature in a systematic way, as well as it covering and measuring the value of the tooth (33). Also, using different tools or reference guides in identifying skin complexion might explain these findings, since other studies relied on visual assessment, but in our case, we used Fitzpatrick skin type to categorize and identify skin complexion.

\section{Conclusions}

The main finding of this study is that there is an inverse relationship between skin color and tooth value among Jordanians. People with fair skin were more likely to have dark teeth (teeth with low value), and people with dark skin color tended to have lighter teeth (teeth with high value). It was concluded that appropriate tooth shade selection has to be customized for each individual in order to make the whole picture mimic nature. In our society, smile designing is highly advocated in esthetic practices, and a perfect integration between facial complexion and teeth is highly important. Yet, further research should be conducted in this field to identify other parameters that could be useful in tooth shade selection. Not to forget other components of esthetic smiles that should be taken into consideration as establishing a healthy functional relationship between oral supporting structures, gingiva and occlusion.

\section{Acknowledgments:}

This study was conducted on patients at dental clinics of different Royal Medical Services - Jordan Armed Forces. We would like to thank our colleagues and staff for their scientific guidance and assistance that helped in improving our work. Also, we are grateful to the reviewers for their valuable comments that helped in improving this version of the manuscript.

\section{Conflict of Interest:}

There is no conflict of interest to be declared.

\section{Authors' contributions:}

All authors contributed to this project and article equally. All authors read and approved the final manuscript.

\section{References:}

1) Dunn WJ, Murchison DF, Broome JC. Esthetics: Patients' Perceptions of Dental Attractiveness. J Prosthodont. 1996; 5(3):166-171. doi: 10.1111/j.1532-849X.1996.tb00292.x. PMID: 9028220.

2) Zarb GA, Bolender C, Hickey JC, Carlsson GE. Boucher's Prosthodontics treatment for edentulous patients. The C.V. Mosby Co. St.Louis; 1990: 338-40.

3) Hassel AJ, Nitschke I, Dreyhaupt J, Wegener I, Rammelsberg P, Hassel JC. Predicting tooth color from facial features and gender: results from a white elderly cohort. J Prosthet Dent. 2008; 99(2): 101-6. doi: 10.1016/S0022-3913(08)60025-6.

4) Sharma V, Punia V, Khandelwal M, Punia S, Lakshmana BR. A study of relationship between skin color and tooth shade value in Population of Udaipur, Rajasthan. International Journal of Dental Clinics. 2010: 2(4): 26-9.

5) Rajtilak G, Deepa S, Rajasekar V, Vanitha R. Anterior Teeth and Smile Designing: A Prospective View. International Journal of Prosthodontics and Restorative Dentistry. 2012; 2(3): 117-27. doi: 10.5005/jpjournals-10019-1060.

6) Preston JD. Colour science and dental art: a self-teaching program. St louis: Mosby; 1980.

7) Goodacre CJ, Sagel PA. Dental esthetics in practice: part 3-understanding colour and shade selection. dentalcare.com-continued $\quad$ education $2011 . \quad$ course. Available from: 
http://www.academia.edu/25017679/Dental_Esthetics_in_Practice_Part_3_-

Understanding Color and Shade Selection.

8) Sabherwal RS, Gonzalez J, Naini FB. Assessing the influence of skin color and tooth shade value on perceived smile attractiveness. J Am Dent Assoc. 2009; 140(6): 696-705.

9) Gozalo-Diaz D, Johnston WM, Wee AG. Estimating the color of maxillary central incisors based on age and gender. J Prosthet Dent. 2008; 100(2): 93-8. doi: 10.1016/S0022-3913(08)60155-9.

10) Jahangiri L, Reinhardt SB, Mehra RV, Matheson PB. Relationship between tooth shade value and skin colour; an observational study. J Prosthet Dent. 2002; 87(2): 149-52. doi: 10.1067/mpr.2002.121109. PMID: 11854669.

11) Esan TA, Olusile AO, Akeredolu PA. Factors influencing tooth shade selection for completely edentulous patients. J Contemp Dent Pract. 2006; 7(5): 80-7. PMID: 17091143.

12) Rasel. Factors affecting skin complexion and texture. 2014. Available from: http://www.bankofinfo.com>how-to-improve-skin.

13) Dummett CO, Sakumura JS, Barens G. The relationship of facial skin complexion to oral mucosa pigmentation and tooth color. J Prosthet Dent. 1980; 43(4): 392-6.

14) Vadavadagi SV, Kumari KV, Choudhury GK, Vilekar AM, Das SS, Jena D, et al. Prevalence of Tooth Shade and its Correlation with Skin Colour - A Cross-sectional Study. J Clin Diagn Res. 2016; 10(2): ZC72-4. doi: 10.7860/JCDR/2016/16918.7324. PMID: 27042590, PMCID: PMC4800657.

15) Al-Dwairi Z, Shaweesh A, Kamkarfar S, Kamkarfar S, Borzabadi-Farahani A, Lynch E. Tooth shade measurements under standard and nonstandard illumination and their agreement with skin color. Int $\mathrm{J}$ Prosthodont. 2014; 27(5): 458-60. doi: 10.11607/ijp.3826. PMID: 25191889.

16) Sample Size Calculator by Raosoft, Inc. Available from: http:/www.raosoft.com/samplesize.html.

17) Bayindir F, Kuo S, Johnston WM, Wee AG. Coverage Error of Three Conceptually Different Shade Guide Systems to Vital Unrestored Dentition. J Prosthet Dent. 2007;98(3):175-185. doi: 10.1016/S00223913(07)60053-5.

18) Creech LL, Mayer JA. Ultraviolet radiation exposure in children: A review of measurement strategies. Ann Behav Med. 1997; 19(9): 399-407.

19) Wikipedia. Fitzpatrick scale. Available from: https://en.wikipedia.org/wiki/Fitzpatrick_scale.

20) Pathak MA. In memory of Thomas Bernhard Fitzpatrick. J Invest Dermatol. 2004; 122: 20-1. doi: 10.1046/j.1523-1747.2003.22248.x. PMID: 15009754.

21) Daniel LC, Heckman CJ, Kloss JD, Manne SL. Comparing Alternative Methods of Measuring Skin Colour and Damage. Cancer Causes Control. 2009; 20(3): 313-321. doi: 10.1007/s10552-008-9245-3. PMID: 18931926, PMCID: PMC2702995.

22) Sachdwa S. Fitzpatrick skin typing: Applications in dermatology. IJDVL. 2009; 75(1): 93-6.

23) Where does your skin fit in? Quiz. Available from: https://www.skincancer.org/prevention/are-you-atrisk/fitzpatrick-skin-quiz.

24) Wikipedia. Ishihara colour test. Available from: https://en.wikipedia.org/wiki/Ishihara_test.

25) Vita tooth guide 3D-Master - VITA North America. Available from: http://www.vitanorthamerica.com.

26) Browning WD, Chan DC, Blalock JS, Brackett MG. A Comparison of Human Raters and an Intra-oral Spectrophotometer. Oper Dent. 2009; 34(3): 337-43. doi: 10.2341/08-106. PMID: 19544824.

27) Sikri VK. Colour: Implications in dentistry. J Conserv Dent. 2010; 13(4): 249-55. doi: 10.4103/09720707.73381. PMID: 21217954, PMCID: PMC3010031.

28) Agrawal VS, Kapoor S. Colour and shade management in esthetic dentistry. 2013; 3(3): 120-7.

29) Rekha SB, Padmasree S, Aprana N, Kumari R. Evaluation of shade Differences between Natural Anterior Teeth in Patients of Different Age Groups, Skin tone, and Gender: A Computerized Cross-sectional Study. International Journal of Scientific study. 2016; 4(1): 231-5.

30) Pichon LC, Landrine H, Corral I, Hao Y, Mayer JA, Hoerster KD. Measuring Cancer Risk in African American: Is Fitzpatrick Skin Type Classification Scale Culturally Sensitive? Ethn Dis. 2010; 20(2): 174-9. PMID: 20503899.

31) Eilers S1, Bach DQ, Gaber R, Blatt H, Guevara Y, Nitsche K, et al. Accuracy of Self-report in Assessing Fitzpatrick Skin Phototypes I Through VI. JAMA Dermatol. 2013; 149(11): 1289-94. doi: 10.1001/jamadermatol.2013.6101. PMID: 24048361.

32) Omari AK, Khammash MR, Matalka I. Skin cancer trends in northern Jordan. Int J Dermatol. 2006; 45 : 384-8. doi: 10.1111/j.1365-4632.2004.02444.x. PMID: 16650163.

33) Smitha AJ, Savitha PN. Shade matching in aesthetic dentistry - from past to recent advances. J Dent Oral Care Med. 2017; 3(1): 102. doi: 10.15744/2454-3276.3.102. 